\title{
Ansiedade social e atribuição de emoções a faces neutras
}

\author{
Nelson Torro Alves \\ Marcelli Roberto Rodrigues \\ Ingrid Brasilino Montenegro Bento de Souza \\ Universidade Federal da Paraiba \\ João Paulo Machado de Sousa \\ Universidade de São Paulo (Ribeirão Preto)
}

\begin{abstract}
Resumo
Trabalhos anteriores têm revelado vieses no reconhecimento de emoções e padrões diferenciais de ativação cerebral no transtorno de ansiedade social. No presente estudo, foi investigada a atribuição de emoções a faces neutras em 22 indivíduos com ansiedade social e 20 voluntários controles. Através do método da escolha forçada, participantes atribuíram emoções de alegria, medo, raiva ou tristeza a faces neutras. Verificou-se que homens e mulheres com ansiedade social atribuíram mais frequentemente emoções de raiva e tristeza às faces neutras, respectivamente. A atribuição de raiva por homens pode estar associada à tendência masculina em detectar sinais de hostilidade no ambiente social, enquanto que o aumento na atribuição de tristeza pelas mulheres pode estar associado à facilitação na identificação de emoções negativas. Os resultados sugerem que a ansiedade social afeta diferentemente os sexos e têm implicações importantes sobre o uso da face neutra como condição de base ou controle nas neurociências comportamentais.
\end{abstract}

Palavras-chave: transtorno de ansiedade social; face neutra; expressões faciais.

\begin{abstract}
Social snxiety and attribution of affect to neutral faces. Previous research has revealed facial emotion recognition biases and distinctive patterns of brain activation in social anxiety disorder. We investigated the attribution of emotion to neutral facial displays in 22 subjects with social anxiety and 20 healthy controls. Using a forced choice paradigm, participants labeled neutral faces as happy, fearful, angry or sad. The most frequent emotional labels attributed by males and females to neutral faces were anger and sadness, respectively. These findings are discussed according to the notion that the attribution of anger by men may be associated with the male tendency to detect hostile environmental signs, whereas the increased attribution of sadness by females might be associated with facilitated identification of negative affect. The results suggest that social anxiety disorder differentially affects males and females and has important implications concerning the use of the neutral face as a baseline or control condition in behavioral neuroscience.
\end{abstract}

Keywords: social anxiety disorder; neutral faces; facial expressions.

$\mathrm{M}$ achado-de-Sousa et al. (2010), em um estudo de revisão da literatura, verificaram que indivíduos com fobia social processam expressões faciais de maneira distinta de voluntários controle, sendo comum a hipervigilância em direção às emoções negativas. Esses achados são confirmados por estudos de neuroimagem funcional, que sugerem que a hipervigilância e a interpretação errônea de expressões faciais estão associadas a alterações nos padrões de conectividade funcional entre as regiões cerebrais, com um aumento na conectividade entre a amígdala e regiões límbicas durante tarefas de avaliação de faces emocionais (Danti et al., 2010). Quando apresentados a faces emocionais de valência negativa, indivíduos socialmente fóbicos mostram maior ativação na amígdala em comparação com voluntários saudáveis (Gentili et al., 2008; Martin, Ressler, Binder, \& Nemeroff, 2010), sendo também observado um aumento na atividade da ínsula durante a visualização de fotografias e faces esquemáticas de raiva (Straube et al., 2004).

A regulação das interações interpessoais depende em grande parte da interpretação correta das expressões faciais (Darwin, 1872/2000; Phillips, Drevets, Raunch, \& Lane, 2003; Trautmann, Fehr, \& Herrmann, 2009). Avaliações imprecisas dos sinais faciais podem interromper o fluxo da conversa, gerar desconforto e levar ao julgamento equivocado de pistas sociais, gerando excesso de reatividade emocional negativa (Goldin et al., 2009; Mullins \& Duke, 2004). Nesse sentido, alterações na percepção das emoções que acompanhem o transtorno de ansiedade social podem prejudicar a adaptação social dos indivíduos.

É importante destacar que o padrão de reconhecimento de expressões faciais pode estar associado ao sexo dos participantes. De maneira geral, mulheres são mais reativas a estímulos emocionais e melhores no reconhecimento das emoções (Whittle, 
Yücel, Yap, \& Allen, 2011). Arrais et al. (2010) verificaram que mulheres em comparação a homens com ansiedade social precisavam de menos intensidade emocional para reconhecer faces de medo, tristeza e alegria.

Os estudos sobre as alterações emocionais na ansiedade social têm focado predominante na precisão da avaliação que esses indivíduos fazem das expressões faciais. No entanto, um número menor de estudos tem explorado os vieses de resposta na atribuição das emoções a faces neutras. Achados de neuroimagem têm mostrado de modo consistente que indivíduos com ansiedade social apresentam um padrão diferencial de ativação cerebral em tarefas que envolvem a avaliação de faces neutras. Cooney et al. (2006), por exemplo, verificaram que, durante a observação de fotografias faces neutras, pacientes com ansiedade social apresentaram uma maior ativação da amígdala direita, enquanto voluntários controles apresentaram uma maior ativação da amígdala esquerda. Esses resultados sugerem que faces neutras eliciam um processamento emocional em ambos os grupos, porém favorecem a hiperatividade no sistema de avaliação emocional negativa em fóbicos sociais, relacionado à ativação da amígdala direita.

Estudos comportamentais indicam que faces neutras podem ser interpretadas de maneira ambígua e que voluntários com fobia social podem lhes atribuir emoções negativas (Winton, Clark, \& Edelmann, 1995; Yoon \& Zinbarg, 2007). Na medida em que "faces neutras" representam uma categoria de estímulo ao menos tão frequente no ambiente social quanto faces emocionais, torna-se especialmente relevante compreender de que maneira possíveis vieses de atribuição das emoções a faces neutras estão relacionadas à ansiedade social.

No presente estudo, foram analisadas respostas comportamentais de atribuição de emoções a faces neutras em homens e mulheres com sintomatologia de ansiedade social e voluntários controle. A análise dos vieses de julgamento dos participantes permitiu verificar que existem padrões diferenciais na atribuição das emoções na ansiedade social e que estão associados ao sexo dos participantes.

\section{Método}

\section{Participantes}

Os participantes foram selecionados por meio da aplicação do Inventário de Fobia Social (Social Phobia Inventory SPIN), validado para o contexto brasileiro por Osório, Crippa e Loureiro (2005). O inventário é composto por 17 itens que avaliam a sintomatologia relacionada ao transtorno de ansiedade social (Connor, Davidson, Churchill, Sherwood, \&Weisler, 2000). Estudos têm revelado bons indicadores de validade e fidedignidade para o SPIN em amostras brasileiras (Osório, Crippa, \& Loureiro, 2010).

Em uma primeira etapa do estudo, o Inventário de Fobia Social foi aplicado a uma amostra de 150 estudantes de graduação da Universidade Federal da Paraíba. A partir da análise das pontuações no inventário foram selecionados para participar do teste de reconhecimento das emoções: a) 22 indivíduos (13 homens e 9 mulheres) com alta sintomatologia relacionada ao transtorno de ansiedade social (escore médio de 26,9 pontos no SPIN) (grupo experimental), e b) outros 20 indivíduos (9 mulheres e 11 homens) com baixa pontuação no inventário de fobia social (escore médio de 10,4 pontos no SPIN) (grupo controle). Os dados da amostra são apresentados na Tabela 1 . $\mathrm{O}$ critério adotado para a definição dos grupos foi baseado no estudo de Osório et al. (2010), que verificaram uma validade discriminante de sensibilidade de 0,86 e especificidade de 0,84 para um corte entre 19 pontos na SPIN.

Foram considerados como critérios de inclusão no estudo: a) idade entre 18 e 30 anos; b) acuidade visual normal ou superior em ambos os olhos, c) ausência de histórico de outros transtornos psiquiátricos, d) uso de drogas e medicamentos neurolépticos. O estudo foi conduzido de acordo com a resolução $n^{0}$ 196/96 do Conselho Nacional de Saúde, que trata da participação de seres humanos em pesquisas, sendo aprovado pelo Comitê de Ética em Pesquisa do Centro de Ciências da Saúde da Universidade Federal da Paraíba, João Pessoa - PB (Protocolo 0164).

Tabela 1

Características demográficas e pontuações obtidas no Inventário de Fobia Social (SPIN) dos grupos do estudo. Os valores são apresentados como média e desvio-padrão.

\begin{tabular}{ccccc}
\hline & \multicolumn{2}{c}{ Grupo Experimental } & \multicolumn{2}{c}{ Grupo Controle } \\
\hline Sexo & $\begin{array}{c}\text { Feminino } \\
(\mathrm{n}=9)\end{array}$ & $\begin{array}{c}\text { Masculino } \\
(\mathrm{n}=13)\end{array}$ & $\begin{array}{c}\text { Feminino } \\
(\mathrm{n}=9)\end{array}$ & $\begin{array}{c}\text { Masculino } \\
(\mathrm{n}=11)\end{array}$ \\
$\begin{array}{l}\text { Idade } \\
\text { (anos) }\end{array}$ & $20,11( \pm 2,33)$ & $20,76( \pm 2,04)$ & $21,66( \pm 2,05)$ & $23,45( \pm 3,93)$ \\
SPIN & $27,3( \pm 7,93)$ & $26,69(+7,94)$ & $11,2( \pm 3,89)$ & $9,81( \pm 4,87)$ \\
\hline
\end{tabular}

\section{Instrumentos}

Para a montagem e aplicação do experimento foi utilizado um computador de modelo Dell Modelo Studio S40 [Intel Core 2 Quad, 250GHz, 4GB], com um monitor Dell (D2201c, 21,5 polegadas), mouse e teclado.

Foram usadas como estímulos experimentais oito fotografias de expressões faciais neutras coloridas de dois modelos masculinos e dois femininos extraídas da série NimStim Emotional Face Stimuli Database (Tottenham et al., 2009).
As fotografias são identificadas na série com os códigos: 37M_NE_C; 37M_NE_O; 41M_NE_C; 41M_NE_O (faces masculinas) e 01F_NE_C; 01F_NE_O; 16F_NE_C; 16_NE_O (faces femininas). Em razão de se haver optado pelo método de escolha forçada na atribuição das emoções, foram também incluídas na sessão experimental expressões faciais de alegria, tristeza, medo e raiva dos mesmos modelos em quatro intensidades emocionais $(25,50,75$ e 100\%). A sequência de apresentação dos estímulos no experimento foi aleatória. Para 
a montagem do experimento foi utilizado o programa SuperLab (versão 4.0).

\section{Procedimento}

A realização do experimento deu-se em sessão individual. Cada participante foi conduzido à sala experimental, aonde era orientado a sentar-se em frente ao computador, ficando posicionado a aproximadamente $70 \mathrm{~cm}$ do centro do monitor.

Antes de iniciar a sessão, os participantes receberam as instruções de que seriam apresentadas expressões de diferentes emoções e intensidades na tela do computador e que, após cada apresentação, deveriam indicar a emoção correspondente à face no teclado numérico do computador. Com o propósito de se atenuar a influência de fatores cognitivos sobre o processo de atribuição das emoções, não foi informado aos participantes que faces neutras seriam apresentadas. Na sessão, cada face era apresentada por 1 segundo, seguida pelo julgamento do participante, que tinha tempo livre de resposta. Após a resposta, uma nova face era apresentada. A Figura 1 ilustra o processo de apresentação de estímulos no experimento.

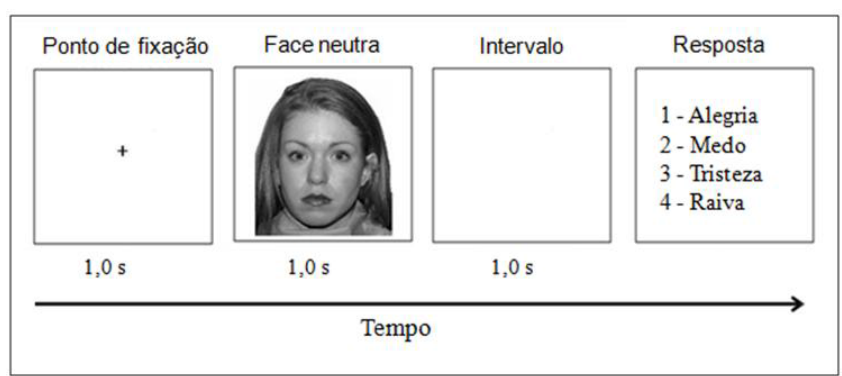

Figura 1. Esquema de apresentação dos estímulos no esperimento

\section{Resultados}

A tabulação dos dados e a análise estatística foram feitas através dos softwares Microsoft Office Excel 2007 e SPSS 18.0. Foram calculadas as frequências de atribuição das emoções às faces neutras. Por ser uma variável categórica, os dados foram submetidos a testes Qui-quadrado $\left(\chi^{2}\right)$ de independência. Foram conduzidas, primeiramente, uma análise geral da atribuição das emoções e, posteriormente, uma análise estatística em separado pelo sexo dos participantes.

\section{Análise geral da atribuição das emoções}

As frequências de atribuição foram submetidas a um teste Qui-quadrado de independência de modelo: 2 grupos (ansiedade social e controle) x 4 emoções (alegria, medo, tristeza e raiva). Foi encontrada uma diferença estatisticamente significativa entre os grupos do estudo (ansiedade social e controle), com o valor do $\chi^{2}$ de 11,22 com um $p<0,01$ para um grau de liberdade de 3 . $\mathrm{O} \mathrm{V}$ de Cramer foi de 0,123 , confirmando a diferença entre os grupos na atribuições das emoções às faces neutras.

Verificou-se que indivíduos com ansiedade social tenderam a atribuir mais frequentemente raiva às faces neutras, enquanto que o grupo controle atribuiu a emoção de medo mais comumente. De maneira geral, a emoção de alegria foi a que obteve a menor frequência de respostas em ambos os grupos. Comparativamente com alegria e raiva, as emoções de medo e tristeza foram mais atribuídas nos julgamentos (Figura 2).

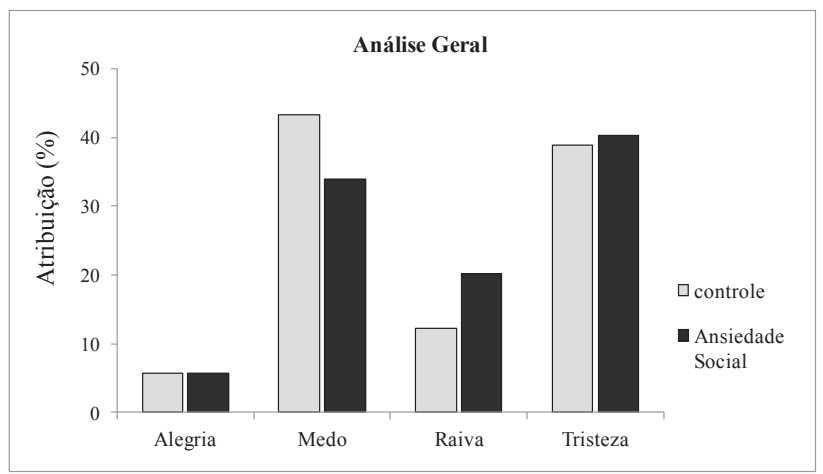

Figura 2. Percentuais gerais de atribuição das emoções de alegria, tristeza, medo e raiva às faces neutras por indivíduos com ansiedade social e voluntários controle

\section{Análise separada dos julgamentos por sexo dos par- ticipantes}

A segunda análise de dados foi realizada com o objetivo de se comparar o efeito da variável sexo do participante sobre a atribuição das emoções. Neste caso, as frequências de atribuições foram submetidas a testes Qui-quadrado em separado por sexo de acordo com o modelo: 2 grupos (ansiedade social e controle) x 4 emoções (alegria, medo, tristeza e raiva).

O Qui-quadrado de independência para o sexo masculino revelou uma diferença estatisticamente significativa entre os grupos de indivíduos com ansiedade social e voluntários controle. Foi encontrado um valor de $\chi^{2}=28,81$, com um $p<$ 0,01 para um grau de liberdade igual a 3 . O teste $\mathrm{V}$ de Cramer apresentou o valor de 0,25 , confirmando a diferença entre os grupos.

De modo semelhante à análise geral, os homens com ansiedade social tiveram uma tendência de atribuir a emoção de raiva às faces neutras. No entanto, vale ressaltar que a magnitude da diferença entre grupo controle e experimental foi consideravelmente maior na amostra composta exclusivamente por homens. Na análise geral, indivíduos com ansiedade social e voluntários controle atribuíram raiva à face neutra em $20,1 \%$ e $12,2 \%$ dos julgamentos, respectivamente, o que corresponde a uma diferença percentual de 7,9\%. Quando considerados apenas os indivíduos do sexo masculino, os percentuais de atribuição dos grupos com ansiedade social e controle foram correspondentes a $28,8 \%$ e $9,4 \%$, respectivamente, totalizando uma diferença de $19,4 \%$.

Tanto na análise geral, quanto na análise dos grupos masculinos, foi encontrada uma atribuição mais frequente de medo às faces neutras pelo grupo controle. Na análise geral, indivíduos com ansiedade social e voluntários controle atribuíram medo, respectivamente, nas frequências de 34,0\% e $43,2 \%$. Considerando-se apenas a amostra masculina, os grupos experimental e controle atribuíram a emoção de medo em 28,4 \% e $41,1 \%$ dos julgamentos, respectivamente. A distribuição das atribuições das emoções é apresentada na Figura 3A. 


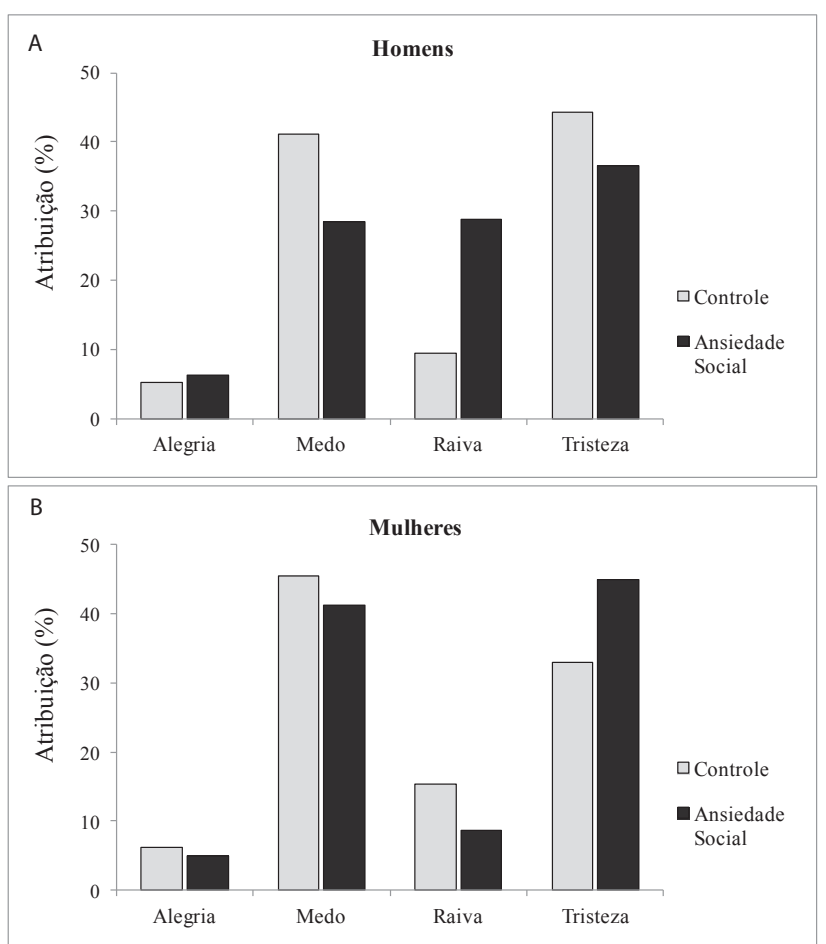

Figura 3. Percentuais de atribuição das emoções de alegria, tristeza, medo e raiva às faces neutras por homens $(\mathrm{A})$ e mulheres $(\mathrm{B})$ com ansiedade social e voluntários controle.

Para a amostra feminina, os resultados do teste $\chi^{2}$ indicaram haver diferenças significativas entre o grupo de mulheres com ansiedade social e voluntárias controle na atribuição das emoções. O valor do $\chi^{2}$ foi de 48,50 com um $p<0,01$ para um grau de liberdade de 3 . O V de Cramer obtido para esses dados foi de 0,38 , confirmando a diferença nas atribuições.

Diferentemente dos resultados encontrados na análise geral e na análise da amostra masculina, verificou-se que mulheres com ansiedade social tiveram a tendência de atribuir mais frequentemente a emoção de tristeza à face neutra em comparação com as voluntárias do grupo controle. Na análise geral, os valores na atribuição da emoção de tristeza foram similares entre os grupos do estudo, com os valores de $38,9 \%$ (controle) e 40,2\% (ansiedade social). Já na análise em separado das mulheres, houve um aumento na magnitude da diferença entre os grupos, passando a figurar a atribuição de tristeza com os valores de $33,0 \%$ e $45 \%$ para os grupos experimental e controle, respectivamente (Figura $3 \mathrm{~B}$ ).

De modo similar à amostra masculina, houve uma menor atribuição da emoção de alegria às faces neutra pelas mulheres dos grupos experimental e controle. De maneira geral, ao longo de todo o estudo, emoções negativas de medo, raiva e tristeza foram atribuídas com maior frequência que a emoção de alegria por homens e mulheres de ambos os grupos experimentais.

\section{Discussão}

O presente estudo utilizou o procedimento de escolha forçada para avaliar a atribuição de emoções de alegria, medo, tristeza e raiva a faces neutras em indivíduos com sintomatologia relacionada ao transtorno de ansiedade social e voluntários controles. Os resultados indicaram que a atribuição das emoções às faces neutras não ocorre de maneira aleatória, mas que determinadas emoções são atribuídas com maior frequência do que outras.

De maneira bastante interessante, verificou-se que a atribuição das emoções depende fortemente da sintomatologia do transtorno de ansiedade social e do gênero do participante. Tanto na análise geral, que contava com o agrupamento dos sexos dos participantes, quanto na análise em separado do sexo masculino, foi observado que os indivíduos com ansiedade social apresentaram um padrão distinto do grupo controle na atribuição das emoções. Indivíduos com ansiedade social tenderam a interpretar faces neutras como expressando raiva. Comparativamente, voluntários do grupo controle atribuíram mais frequentemente a emoção de medo às faces. Tais resultados são concordantes com achados na literatura, que revelam a existência de alterações no reconhecimento de emoções faciais em indivíduos com o transtorno de ansiedade social (Machadode-Sousa et al., 2010).

Em geral, estudos têm revelado que portadores do transtorno apresentam um padrão de hipervigilância/evitação para estímulos sociais potencialmente negativos. Horley et al. (2004) em um estudo de registro dos movimentos oculares, verificaram que indivíduos com ansiedade social apresentam menos fixações nas regiões dos olhos, ao mesmo tempo em que executam maior varredura visual de outras regiões do rosto em comparação com voluntários controles, particularmente durante a observação de faces de raiva. Presume-se que a evitação da região dos olhos esteja associada ao medo subjacente da avaliação negativa nas interações sociais, enquanto que a hipervarredura da face é consistente com a ideia de que estímulos ameaçadores devem ser rapidamente detectados e processados. Schneier et al. (2011) verificaram que a severidade dos sintomas na ansiedade social estava associada com a evitação do olhar nas interações sociais, avaliada por meio da aplicação de um instrumento de autorrelato (Gaze Anxiety Rating Scale - GARS). Após o período de 8 a 12 semanas de tratamento com a administração de Paroxetina houve uma diminuição da evitação do olhar no grupo com ansiedade social.

Os resultados obtidos para os homens sustentam a hipótese de que indivíduos com ansiedade social apresentam uma hipervigilância para a detecção de sinais de ameaça no ambiente social, o que se confirma pela atribuição da emoção de raiva às faces neutras. De acordo com o modelo cognitivo de ansiedade social, indivíduos com o transtorno de ansiedade social supõem que serão negativamente avaliados em um contexto social e concentram seus recursos atencionais nessas representações mentais, interpretando de modo impreciso as pistas sociais (Rapee \& Heimberg, 1997).

Para o grupo de mulheres do presente estudo, houve um padrão distinto na atribuição das emoções. Mulheres com ansiedade social consideraram que faces neutras expressavam tristeza com maior frequência que as voluntárias controles. Tais diferenças encontradas entre os indivíduos com ansiedade social do sexo masculino e feminino podem estar associadas 
às diferenças subjacentes entre os sexos, que existem nos domínios da anatomia, química e funções cerebrais (Cahill, 2006). No julgamento de expressões faciais, homens apresentam relativamente maior dificuldade para discriminar as emoções em comparação às mulheres (Montagne, Kessels, Frigerio, de Haan, $\&$ Perrett, 2005). Mulheres também detectam mais facilmente expressões positivas, como alegria (Biele \& Grabowska, 2006).

Dessa maneira, pode-se aventar a hipótese de que a ansiedade social promove respostas diferentes em homens e mulheres e que estão associadas ao quadro psicopatológico de fundo. A atribuição de tristeza mais frequente pode estar relacionada à predisposição encontrada em mulheres para o desenvolvimento da depressão (Rutter, Caspi, \& Moffitt, 2003), cuja prevalência durante a vida é duas vezes maior nas mulheres do que nos homens (Kaplan, Sadock, \& Grebb, 2003). Não pode ser descartada também a possibilidade de que mulheres com ansiedade social tenham mais receio de entristecer ou decepcionar as outras pessoas, ao invés de provocar a raiva, o que poderia ser responsável pelas diferenças entre os sexos na atribuição das emoções. Estudos de socialização parental mostram que pais se relacionam de maneira distinta dependendo do sexo de seus filhos (Cassano \& Zeman, 2010). Em geral, os pais conversam com mais frequência com as meninas sobre emoções de tristeza (Adams, Kuebli, Boyle, \& Fivush, 1995) e desestimulam a expressão aberta desse sentimento entre os meninos (Chaplin, Cole, \& Zahn-Waxler, 2005). Estudos de neuroimagem confirmam diferenças nos padrões de ativação cerebral, mostrando que as mulheres, em comparação aos homens, apresentam mais atividade nas estruturas límbicas anteriores (cingulado anterior, caudado, córtex pré-frontal medial) durante a experiência emocional de tristeza (George, Ketter, Parekh, Herscovitch, \& Post, 1996; Whittle et al., 2011).

Uma limitação do presente estudo foi a não aplicação de um instrumento de avaliação da depressão nos participantes, o que permitiria determinar se o viés de julgamento das mulheres está mais associado aos sintomas da depressão ou da ansiedade social. Tal problema poderia ser solucionado com o registro de uma medida de depressão e sua inclusão como uma covariável estatística. Hunter, Buckner e Schmidt (2009), em um estudo realizado sobre o reconhecimento de expressões emocionais (alegria, tristeza, medo e raiva), verificaram que participantes com altos índices de ansiedade social também apresentavam mais sintomas de depressão. No entanto, foi possível aos autores isolar o efeito da ansiedade ao incluírem a depressão como uma das covariáveis que influencia o reconhecimento das emoções. Futuras investigações sobre a atribuição de emoções a faces neutras poderão analisar de modo mais categórico o papel da sintomatologia depressiva nos julgamentos. Deve-se ressaltar que é recomendada a aplicação de uma escala sintomática mesmo na ausência de um diagnóstico clínico de depressão, tendo em vista que é possível que sintomas leves de depressão influenciem os padrões de respostas.

Os resultados do presente estudo devem ser também analisados no contexto dos atuais questionamentos acerca do uso de expressões faciais estáticas em estudos do reconhecimento de emoções. Alguns autores têm atribuído menor validade ecológica a faces estáticas, dado o fato de que nas interações do dia a dia é necessária a decodificação de aspectos temporais do movimento facial (Arsalidou, Morris, \& Taylor, 2011). Em complementação, tem sido demonstrado que expressões dinâmicas são em geral mais bem reconhecidas que expressões estáticas (Ambadar, Schooler, \& Cohn, 2005) e evocam respostas mais intensas de ativação fisiológica e mímica facial (Sato, Fujimura, \& Suzuki, 2008). A presente investigação, no entanto, insere-se em um âmbito ligeiramente distinto dos trabalhos mencionados, na medida em que foi analisada a atribuição de emoções a faces neutras e não o reconhecimento das emoções.

Uma importante conclusão é a de que "faces neutras", em função dos vieses de atribuição encontrados, não podem ser consideradas absolutamente neutras. De fato, faces neutras podem, inclusive, conter elementos estruturais que as tornam semelhantes a faces emocionalmente expressivas. Said, Todorov e Sebe (2009) verificaram, por exemplo, que faces neutras percebidas com valência positiva se assemelhavam a faces de alegria, faces consideradas com valência negativa se assemelhavam as expressões de nojo e medo e faces neutras percebidas como ameaçadores relembravam raiva. Dessa maneira, pode-se considerar que faces neutras são capazes de evocar respostas emocionais nos participantes. Tais achados são especialmente relevantes, tendo em vista o uso de faces neutras como linha de base ou condição controle em estudos de neuroimagem.

Em resumo, os achados do presente trabalho indicam que a ansiedade social promove vieses de atribuição de emoções a faces neutras que estão associados ao sexo dos indivíduos. De maneira geral, homens com a sintomatologia tendem a interpretar faces neutras como expressando raiva, enquanto as mulheres as percebem como tristeza. Os resultados indicam que a atribuição de emoções a faces neutras pode representar um importante instrumento de avaliação das alterações emocionais presentes no transtorno de ansiedade social, podendo ser também empregado no estudo de outros transtornos psiquiátricos, como a depressão, o transtorno do pânico e a esquizofrenia. Por fim, a investigação dos vieses de interpretação da face neutra pode auxiliar o desenvolvimento de condições de base ou controle mais apropriadas no campo das neurociências cognitivas.

\section{Referências}

Adams, S., Kuebli, J., Boyle, P., \& Fivush, R. (1995). Gender differences in parent-child conversations about past emotions: a longitudinal investigation. Sex Roles, 33, 309-323.

Ambadar, Z., Schooler, J., \& Cohn, J. F. (2005). Deciphering the enigmatic face: the importance of facial dynamics to interpreting subtle facial expressions. Psychological Science, 16, 403-410.

Arrais, K. C., Machado-de-Sousa, J. P., Trzesniak, C., Santos Filho, A., Ferrari, M. C., Osorio, F. L., \& Crippa, J. A. (2010). Social anxiety disorder women easily recognize fearfull, sad and happy faces: the influence of gender. Journal of Psychiatric Research, 44(8), 535-540.

Arsalidou, M., Morris, D., \& Taylor, M. J. (2011). Converging evidence for the advantage of dynamic facial expressions. Brain Topography, 24(2), 149-163.

Biele, C., \& Grabowska, A. (2006). Sex differences in perception of emotion intensity in dynamic and static facial expressions. Experimental Brain Research, 171, 1-6

Cahill, L. (2006). Why sex matters for neuroscience. Nature Reviews 
Neuroscience, 7(6), 477-484.

Cassano, M. C., \& Zeman, J. L. (2010). Parental socialization of sadness regulation in middle childhood: the role of expectations and gender. Developmental Psychology, 46(5), 1214-1226.

Chaplin, T. M., Cole, P. M., \& Zahn-Waxler, C. (2005). Parental socialization of emotion expression, gender differences, and relations to child adjustment. Emotion, 5, 80-88.

Connor, K. M., Davidson, J. R. T., Churchill, E. L., Sherwood, A., \& Weisler, R. H. (2000). Psychometric properties of the social phobia Invenctory (SPIN). The British Journal of Psychiatry, 176, 379-386.

Cooney, R. E., Atlas, L. Y., Jooormann, J., Eugéne, F., \& Gotlib, I. H. (2006). Amygdala activation in the processing of neutral faces in social anxiety disorder: is neutral really neutral? Psychiatry Research, 148(1), 55-59.

Danti, S., Ricciardi, E., Gentili, C., Gobbino, M. I., Pietrini, P., \& Guazzelli, M. (2010). Is a Social phobia a "mis-communication" disorder? Brain functional connectivity during face perception differs between patients with social phobia and healthy control subjects. Frontiers in Systems Neuroscience, 152(4), 1-11.

Darwin, C. A. (2000). A expressão das emoções no homem e nos animais. $\left(2^{\mathrm{a}}\right.$ ed., L. S. L. Garcia, Trad.). São Paulo: Cia das Letras. (Texto original publicado em 1872).

Gentili, C., Gobbini, M. I., Ricciardi, E., Vanello, N., Pietrini, P., Haxby, J. V., \& Guazzelli, M. (2008). Diferencial modulation of neural activity throughout the distributed neural system for face perception in patients with social phobia and healthy subjects. Brain Research Bulletin, 77(5), 286-292.

George, M. S., Ketter, T. A., Parekh, P. I., Herscovitch, P., \& Post, R. M. (1996). Gender differences in regional cerebral during transient self-induced sadness or blood flow happiness. Biological Psychiatry, 40, 859-871.

Goldin, P. R., Manber, T., Hakimi, S., Canli, T., \& Gross, J. J. (2009). Neural bases of social anxiety disorder: emotional reactivity and cognitive regulation during social and physical threat. Archives of General Psychiatry, 66(2), 170-180.

Horley, K., Williams, L. M., Gonsalvez, C., \& Gordon, E. (2004). Face to face: visual scanpath evidence for abnormal processing of facial expressions in social phobia. Psychiatry Research, 127(1), 43-53.

Hunter, L. R., Buckner, J. D., \& Schmidt, N. B. (2009). Interpreting facial expressions: the influence of social anxiety, emotional valence, and race. Journal of Anxiety Disorders, 23(4), 482-488.

Kaplan, H. B., Sadock, B. J., \& Grebb, J. A. (2003). Compêndio de psiquiatria: Ciências do comportamento e psiquiatria clínica. Porto Alegre: Artes Médicas.

Machado-de-Sousa, J. P., Arrais, K. C., Alves, N. T., Chagas, M. H., de MenesesGaya, C., Crippa, J. A., \& Hallak, J. E. (2010). Facial affect processing in social anxiety: tasks and stimuli. Journal of Neuroscience Methods, 193(1), $1-6$.

Martin, E. I., Ressler, K. J., Binder, E., \& Nemeroff, C. B. (2010). The neurobiology of anxiety disorders: brain imaging, genetics, and psychoneuroendocrinology. Psychiatric Clinics of North America, 30, 865-891.

Montagne, B., Kessels, R. P., Frigerio, E., de Haan, E. H., \& Perrett, D. I. (2005). Sex differences in the perception of affective facial expressions: do men really lack emotional sensitivity? Cognitive Processing, 6(2), 136-41.

Mullins, D. T., \& Duke, M. P. (2004). Effects of social anxiety on nonverbal accuracy and response time I: facial expressions. Journal of nonverbal behavior, 28(1), 3-33.

Osório, F. L.; Crippa, J. A. S.; Loureiro, S. R. (2005). Instrumentos de avaliação do transtorno de ansiedade social. Revista Psiquiatria Clínica, 32(2) 73-83.

Osório, F. L., Crippa, J. A. S., Loureiro, S. R. (2010). Evaluation of the psychometric properties of the Social Phobia Inventory in university students. Comprehensive Psychiatry, 51, 630-640.

Phillips, M. L., Drevets, W. C., Raunch, S. L., \& Lane, R. (2003). Neurobiology of emotion perception I: the neural basis of normal emotion perception. Biological Psychiatry, 505(54), 504-514.

Rapee, R. M., \& Heimberg, R. G. (1997). A cognitive-behavioral model of anxiety in social phobia. Behavior Research and Therapy, 35, 741-756.

Rutter, M., Caspi, A., \& Moffitt, T. E. (2003). Using sex differences in psychopathology to study casual mechanisms: unifying issues research strategies. Journal of Child Psychology and Psychiatry, 44(8), 1092-1115.

Said, C., Todorov, A., \& Sebe, N. (2009). Structural resemblance to emotional expressions predicts evaluation of emotionally neutral faces, America Psychological Association, 9(2), 260-264.

Sato, W., Fujimura, T., \& Suzuki, N. (2008). Enhanced facial EMG activity in response to dynamic facial expressions. International Journal of Psychophysiology, 70(1), 70-74.

Schneier, F. S., Rodebaugh, T. L., Blanco, C., Lewin, H., \& Liebowitz, M. R. (2011). Fear and avoidance of eye contact in social anxiety disorder. Comprehesive Psychiatry, 52(1), 81-87.

Straube, T., Kolassa, I. T., Glauer, M., Mentzel, H. J., \& Miltner, W. H. (2004). Effect of task conditions on brain responses to threatening faces in social phobics: an event-related functional magnetic resonance imaging study. Biological psychiatry, 56(12), 921-930.

Trautmann, S. A., Fehr, T., \& Herrmann, M. (2009). Emotion in motion: dynamic compared to static facial expression of disgust and happiness reveal more widespread emotion-specific activations. Brain Research, 1284, 100-115.

Whittle, S., Yücel, M., Yap, M. B. H., \& Allen, N. B. (2011). Sex differences in the neural correlates of emotion: evidence from neuroimaging. Biological Psychology, 87, 319-333.

Winton, E. C., Clark, D. M., \& Edelmann, R. J. (1995). Social anxiety, fear of negative evaluation and the detection of negative emotion in others. Behaviour Research and Therapy, 33(2), 193-196.

Yoon, K. L., \& Zinbarg, R. E. (2008). Interpreting neutral faces as threatening is a default mode for socially anxious individuals. Journal of abnormal psychology, 117(3), 680-685.

Nelson Torro Alves, doutor em Psicobiologia pela Universidade de São Paulo - Ribeirão Preto, é professor Adjunto na Universidade Federal da Paraíba. Telefones: (83) 9111-0423/3216-7337. Fax: (83) 3216-7064. Email: nelsontorro@yahoo.com.br

Marcelli Roberto Rodrigues, graduada em Psicologia pela Universidade Federal da Paraíba. Email: marcellirr@hotmail.com

Ingrid Brasilino Montenegro Bento de Souza, graduanda em Psicologia na Universidade Federal da Paraíba. Email: ingrid_brasilino@hotmail.com

João Paulo Machado de Sousa, doutor em Saúde Mental pela Universidade de São Paulo - Ribeirão Preto. Email:.jpmpaulos@yahoo.com.br 